Pathologe 2016 · 37:434-440

DOI 10.1007/s00292-016-0198-0

Online publiziert: 9. August 2016

(C) Der/die Autor(en) 2016. Dieser Artikel ist eine Open-Access-Publikation.

Schwerpunktherausgeber

A. Marx, Mannheim

P. Ströbel, Göttingen

CrossMark

\section{Neuroendokrine Tumoren (NET) können in verschiedenen Organen auftreten. Allen gemeinsam sind ihre neuroendokrine Morphologie und die Expression neuroendokriner Marker. Die diagnostischen Kriterien, das Grading sowie die Nomenklatur dieser Neoplasien variieren je nach Organ. Universell ist der Zusammenhang zwischen steigender biologischer Tumoraggressivität und höherer Mitoserate. NET des Mediastinums sind selten, zeigen aber meist klassische klinische Symptome. Eine einheitliche Klassifikation, Stadieneinteilung und Therapie stellen auch heute noch kontrovers diskutierte Punkte dar.}

\section{Hintergrund}

Das Mediastinum findet sich anatomisch zwischen linker und rechter Lunge, in sagittaler Ausbreitung zwischen Sternum und Wirbelsäule, kranial willkürlich begrenzt durch die Apertura thoracica superior und kaudal begrenzt durch das Zwerchfell. Es stellt einen größeren Anteil des Thorax dar und beinhaltet zahlreiche Organe und Strukturen. Während der Embryonalentwicklung dient es als Migrationspfad pluripotenter Stammzellen und Organe. Aus all diesen Strukturen können sich Neoplasien entwickeln, wodurch sich ein weites Spektrum maligner und benigner Tumoren ergibt. Primäre neuroendokrine Tumoren sind sehr selten, wobei NET des Thymus die größte Gruppe darstellen. In der Summe machen sie $5 \%$ aller Tumoren des Mediastinums und des Thymus aus und treten

L. Brcic $\cdot$ M. Heidinger $\cdot H$. Popper

Institut für Pathologie, Medizinische Universität Graz, Graz, Österreich

\title{
Neuroendokrine Neoplasien des Mediastinums
}

vorrangig im vorderen Mediastinum auf $[13,15,24]$.

\section{Klassifikationen}

Kontroversen und Unsicherheit bestehen auch heute noch bzgl. der Klassifikation von NET. In der vorletzten Klassifikation der WHO aus dem Jahr 2004 wurden sie in gut differenzierte neuroendokrine Karzinome (typische und atypische Karzinoide) und schlecht differenzierte neuroendokrine Karzinome (großzellige neuroendokrine Karzinome und kleinzellige Karzinome) unterteilt [30]. In der letzten WHO-Klassifikation von 2015 wurden alle neuroendokrinen Karzinome in 3 Gruppen unterteilt (• Tab. 1):

- niedriggradig maligne NET (typisches Karzinoid),

- mäßiggradig maligne NET (atypisches Karzinoid) und

- hochgradig maligne NET (großzelliges neuroendokrines Karzinom, kleinzelliges Karzinom).

Die neue WHO-Klassifikation der thymischen NET hat bei der Einteilungsänderung der mediastinalen/thymischen NET in 3 Malignitätsgrade die Nomenklatur an die der Lunge angepasst, während die histologischen Kriterien der eigentlichen Entitäten gleich geblieben sind. Kombinierte Tumoren, welche klein- oder großzellige neuroendokrine Karzinome enthalten, sollten als kombiniertes kleinzelliges Karzinom bzw. kombiniertes großzelliges neuroendokrines Karzinom diagnostiziert werden [29].

Ein weiteres Problem ist die unterschiedliche Klassifikation der NET im Gastrointestinaltrakt (GI-NET), wobei von der Klassifikationsgruppe versucht wurde, diese Klassifikation auch auf die NET der Lunge zu übertragen (für das Mediastinum existieren derzeit noch $\mathrm{zu}$ wenige Daten). Dieser Versuch ist aber fehlgeschlagen, vor allem, weil die Biologie der pulmonalen (und möglicherweise mediastinalen) Karzinoide möglicherweise nicht direkt vergleichbar mit GINET ist. Bei den atypischen Karzinoiden der Lunge finden sich genetische Veränderungen, die auf Zigarettenrauch zurückzuführen sind (z. B. Punktmutationen in TP53) und zumeist einen negativen Einfluss auf die Prognose haben. Tumoren mit der Morphologie atypischer Karzinoide sind im Gastrointestinaltrakt eher selten, auch die hochmalignen neuroendokrinen Karzinome treten in der Lunge deutlich häufiger auf. Die derzeitigen Grenzwerte für die Mitoseraten mediastinaler NET haben sich als prognostisch valide erwiesen [25]. Der für die Unterscheidung zwischen NET-G2 und NEC angesetzte Cut-off-Wert von 20 Mitosen/10 „high power field“ (HPF) ist für mediastinale NET möglicherweise zu hoch angesetzt.

\section{Klinische Präsentation}

Großteils treten NET des Thymus im anterioren/anteriosuperioren Mediastinum bei Erwachsenen zwischen 40 und 60 Jahren auf. In erster Linie sind Männer betroffen, wobei dieser Zusammenhang am stärksten bei Karzinoiden, bei großzelligen neuroendokrinen Karzinomen (LCNEC) bereits weniger stark und bei kleinzelligen Karzinomen (SCC) nicht mehr ausgeprägt ist. Bei Letzterem sind beide Geschlechter gleich häufig betroffen $[1,3,24,25]$. 
Tab. 1 WHO-Klassifikationen neuroendokriner Tumoren des Thymus

\begin{tabular}{|c|c|c|}
\hline & WHO 2004 & WHO 2015 \\
\hline \multirow[t]{3}{*}{ TC } & Gut differenzierte NET & Niedriggradig maligne NET \\
\hline & $<2$ Mitosen $/ 2 \mathrm{~mm}^{2}$ & $<2$ Mitosen $/ 2 \mathrm{~mm}^{2}$ \\
\hline & Keine Nekrosen & Keine Nekrosen \\
\hline \multirow[t]{3}{*}{ AC } & Gut differenzierte NET & Mäßiggradig maligne NET \\
\hline & $2-10$ Mitosen $/ 2 \mathrm{~mm}^{2}$ & $2-10$ Mitosen $/ 2 \mathrm{~mm}^{2}$ \\
\hline & Nekrosen bei 0-10 Mitosen $/ 2 \mathrm{~mm}^{2}$ & Nekrosen bei 0-10 Mitosen $/ 2 \mathrm{~mm}^{2}$ \\
\hline \multirow[t]{4}{*}{ LCNEC } & Gering differenzierte NET & Hochgradig maligne NET \\
\hline & Nichtkleinzellige NET & Nichtkleinzellige NET \\
\hline & $>10$ Mitosen $/ 2 \mathrm{~mm}^{2}$ & $>10$ Mitosen $/ 2 \mathrm{~mm}^{2}$ \\
\hline & Nekrosen (häufig, aber nicht obligat) & Nekrosen (häufig, aber nicht obligat) \\
\hline \multirow[t]{4}{*}{ SCC } & Gering differenzierte NET & Hochgradig maligne NET \\
\hline & Kleinzellige Zytologie & Kleinzellige Zytologie \\
\hline & $>10$ Mitosen $/ 2 \mathrm{~mm}^{2}$ & $>10$ Mitosen $/ 2 \mathrm{~mm}^{2}$ \\
\hline & Nekrosen (häufig, aber nicht obligat) & Nekrosen (häufig, aber nicht obligat) \\
\hline \multicolumn{3}{|c|}{$\begin{array}{l}\text { TC typisches Karzinoid, AC atypisches Karzinoid, LCNEC großzelliges neuroendokrines Karzinom, } \\
\text { SCC kleinzelliges Karzinom, NET neuroendokriner Tumor }\end{array}$} \\
\hline
\end{tabular}

Hier steht eine Anzeige. Springer
Bei bis zu einem Drittel präsentieren sich die Tumoren asymptomatisch [4] und stellen Zufallsbefunde bei Untersuchungen wegen anderer Indikationen dar. Vorliegende Symptome resultieren typischerweise durch lokales $\mathrm{Tu}$ morwachstum, Fernmetastasen und/ oder endokrine Symptomatik. Lokales Wachstum äußert sich durch Dyspnoe, thorakalen Druckschmerz, Husten und obere Einflussstauung (Vena-cava-superior-Syndrome). Gewichtsabnahme wurde bei manchen Patienten beschrieben. Endokrine Symptomatik zeigt sich als Cushing-Syndrom, Hyperkalziämie/ Hypophosphatämie, Überproduktion von Adiuretin $(\mathrm{ADH})$, seltener Akromegalie und als Karzinoidsyndrom. NET können auch im Zuge des Multiple-endokrine-Neoplasie-1(MEN1)-Syndroms (nur Karzinoide und nur Männer) auftreten $[14,26]$ und stehen in Assoziation zum Eaton-Lambert-Syndrom und zur hypertrophen Osteoarthropathie [2].

\section{Makroskopie}

NET des Thymus präsentieren sich einerseits als kleine, aber praktisch immer funktionelle (bis zu minimal $1,3 \mathrm{~cm}$; [32]), andererseits aber bis zu $20 \mathrm{~cm}$ große Tumoren $[21,24,30]$. Sie können bekapselt und umschrieben vorliegen, besitzen meist allerdings keine Kapsel und dringen in Pleura, Perikard, Lunge oder
Adventitia der großen Gefäße ein. Die Schnittfläche präsentiert sich druckfest, grauweiß bis bräunlich und ohne die charakteristische Lobulierung von Thymomen. In bis zu $30 \%$ der Fälle zeigen die Tumoren Kalzifizierungen. Die Tumoren können Einblutungen und Nekrosezonen aufweisen, wobei dies v. a. in kleinzelligen Karzinomen auftritt.

\section{Typische Karzinoide}

Typische Karzinoide sind definiert als neuroendokrine epitheliale Tumoren des Thymus, mit weniger als 2 Mitosen $/ 2 \mathrm{~mm}^{2}$ (in $10 \mathrm{HPF}$ ) ohne Nekrosen. Sie umfassen etwa $20 \%$ aller NET des Thymus und treten häufiger bei Männern auf (mehr als zwei Drittel der Patienten mit typischen Karzinoiden sind Männer). Das durchschnittliche Alter bei Diagnose beträgt 49 Jahre [24], bei Patienten mit MEN1-Syndrom 44 Jahre [27]. Patienten mit MEN1 entwickeln in $8 \%$ der Fälle Karzinoide des Thymus [14] wobei nur bei Männern Rauchen als Risikofaktor besteht $[14,16]$.

Etwa die Hälfte aller Patienten präsentiert sich mit Symptomen des lokalen Tumorwachstums - Husten, Dyspnoe, Druckschmerz und Einflussstauung [15, 24]. Bei bis zu einem Drittel der erwachsenen Patienten sowie bei mehr als der Hälfte von Kindern mit typischen Karzinoiden entwickelt sich 
ein Cushing-Syndrom mit oder ohne Hyperpigmentation der Haut [12]. Durch Hyperparathyreoidismus im $\mathrm{Zu}$ ge des MEN1 oder der Parathyroid-hormone-related-Proteinsekretion des $\mathrm{Tu}$ mors kann es in weiterer Folge zur Hyperkalziämie/Hypophosphatämie kommen [33]. Akromegalie, Karzinoidsyndrom sowie synchron auftretende maligne Prozesse können vorkommen, stellen aber seltene Ereignisse dar. Im Verlauf der Erkrankung entwickeln bis zu $50 \%$ der Patienten regionale Lymphknotenmetastasen oder Fernmetastasen in Lunge, Knochen oder anderen Organen [24].

\section{Histologie}

Histologisch präsentieren sich die Karzinoide mit kleinen bis mittelgroßen, uniform polygonalen Zellen mit runden Kernen und feinkörnigem Chromatin sowie kleinen unauffälligen Nukleoli. Das Zytoplasma ist blass-basophil oder eosinophil. Koagulationsnekrosen und/oder mehr als 2 Mitosen/ $2 \mathrm{~mm}^{2}$ stellen Ausschlusskriterien für typische Karzinoide dar. Typisch und diagnostisch sind organoide Muster, Rosetten und Nester mit nukleärem Palisadenmuster (• Abb. 1). Es können auch solide sowie pseudoglanduläre Muster vorkommen. Häufig kommt es zu einer Lymphgefäßinvasion. Beide, typische und atypische Karzinoide haben mehrere morphologische Varianten wie spindelzellige, pigmentierte, amyloidhaltige, onkozytäre, muzinöse, angiomatöse und chondroide Ausformungen [29].

\section{Immunhistochemie}

Immunhistochemisch präsentieren sich typische Karzinoide in einem punktförmigen Färbungsmuster positiv für Zytokeratin AE/AE3 und CAM 5.2 sowie für neuroendokrine Marker wie CD56 (NCAM), Synaptophysin, Chromogranin und neuronenspezifische Enolase (NSE). NCAM kann in typischen Karzinoiden negativ sein, nimmt an Färbeintensität typischerweise in den höhergradigen NET deutlich zu (der Grund sind unterschiedliche Splicevarianten des Proteins, wo die 140-KDaForm charakteristisch für das kleinzellige Karzinom ist und mit dem am Häufigsten verwendeten Antikörper erfasst wird).

Pathologe 2016 -37:434-440 DOI 10.1007/s00292-016-0198-0

(c) Der/die Autor(en) 2016. Dieser Artikel ist eine Open-Access-Publikation.

\section{Brcic $\cdot$ M. Heidinger $\cdot$ H. Popper}

\section{Neuroendokrine Neoplasien des Mediastinums}

\section{Zusammenfassung}

Primäre neuroendokrine Tumoren (NET) des Mediastinums sind selten, wobei neuroendokrine Neoplasien des Thymus die häufigsten darstellen. Insgesamt machen sie $5 \%$ aller Tumoren des Mediastinums und Thymus aus. Die WHO-Klassifikation aus dem Jahr 2015 unterteilt NET des Thymus in 3 Gruppen: niedriggradig maligne (typisches Karzinoid), mäßiggradig maligne (atypisches Karzinoid) und hochgradig maligne NET (großzelliges neuroendokrines Karzinom, kleinzelliges Karzinom). Bei dieser Einteilungsänderung der mediastinalen/thymischen NET in 3 Malignitätsgrade wurde die Nomenklatur an die der Lunge angepasst, während die histologischen Kriterien der eigentlichen Entitäten gleich geblieben sind. NET treten typischerweise bei Erwachsenen mittleren Alters, vorwiegend bei Männern auf. Etwa $30 \%$ sind asymptomatisch, wobei die restlichen durch Symptomatik des lokalen Tumorwachstums, Fernmetastasen und/oder endokrine Symptomatik gekennzeichnet sind.
Karzinoide können auch als Teil des MEN1Syndroms (multiple endokrine Neoplasie) auftreten. Zum Zeitpunkt der Diagnose präsentieren sie sich häufig mit regionalen Lymphknoten oder Fernmetastasen, wobei Lunge und Knochen die primären Absiedelungsgebiete darstellen. Die wesentlichen Parameter der Diagnostik neuroendokriner Tumoren umfassen die Zellmorphologie sowie das Vorliegen und die Anzahl von Mitosen und/oder Nekrosen. Patienten mit typischen Karzinoiden zeigen die beste Prognose, während sie bei atypischen Karzinoiden sowie großzelligen neuroendokrinen Tumoren bereits schlechter ist und kleinzellige Karzinome mit einem durchschnittlichen Überleben von 14 Monaten die schlechteste Prognose aufweisen.

Schlüsselwörter

Klassifikation · Karzinoide - MEN1-Syndrom . Zellmorphologie $\cdot$ Prognose

\section{Neuroendocrine neoplasms of the mediastinum}

\section{Abstract}

Primary neuroendocrine tumors (NET) in the mediastinum are very rare and among them thymic NETs are the most common. They represent $5 \%$ of all thymic and mediastinal tumors. The WHO classification from 2015 subdivides thymic NETs into three groups; low grade (typical carcinoid), intermediate grade (atypical carcinoid) and high grade (large cell neuroendocrine carcinoma and small cell carcinoma). Through this change of mediastinal/thymic NET classification into three groups of malignancy, the nomenclature was adapted to that of the lungs, while the histological criteria for each entity remained the same. Thymic NETs typically occur in middle-aged adults and predominantly in males. Approximately $30 \%$ are asymptomatic and the rest present with symptoms caused by local tumor growth, distant metastases and/or endocrine manifestations. Carcinoids can also occur as a part of multiple endocrine neoplasia type 1 (MEN1) and at the time of diagnosis commonly present with regional lymph node or distant metastases, which most often affect the lungs and bones. For the correct diagnosis tumor cell morphology, mitotic count and/or necrosis are crucial. Patients with typical carcinoids have the best prognosis, whereas the prognosis is slightly worse for atypical carcinoids but very poor for large cell neuroendocrine carcinomas. Small cell carcinomas have the worst prognosis and the shortest median survival time of approximately 14 months.

\section{Keywords}

Classification · Carcinoids · MEN 1 - Cell morphology $\cdot$ Prognosis
Der thyroidale Transkriptionsfaktor-1 (TTF1) ist typischerweise negativ [23]. In einzelnen Tumorzellen sind einige Hormone wie das adrenokortikotrope Hormon (ACTH), Calcitonin, Somatostatin oder humanes Choriongonadotropin (HCG) typischerweise immun- histochemisch positiv, wobei sich keine Korrelation zur klinischen Präsentation ergibt [1].

\section{Differenzialdiagnostik}

Die Differenzialdiagnostik umfasst $\mathrm{Pa}$ ragangliome, die meistens histologisch 

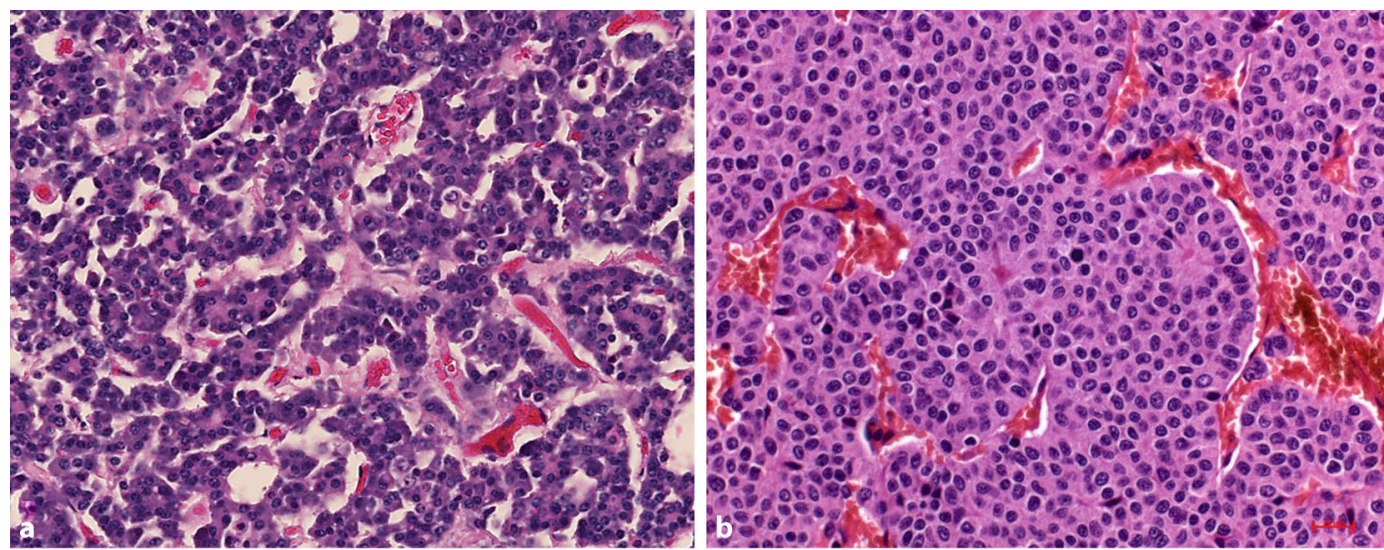

Abb. $1 \varangle$ Histologische Präsentation eines neuroendokrinen Tumors des Thymus. Typisches Karzinoid (a) mit tubulären und azinären Mustern sowie Pseudorosetten. Atypisches Karzinoid (b) mit typischer neuroendokriner Morphologie, organoidem
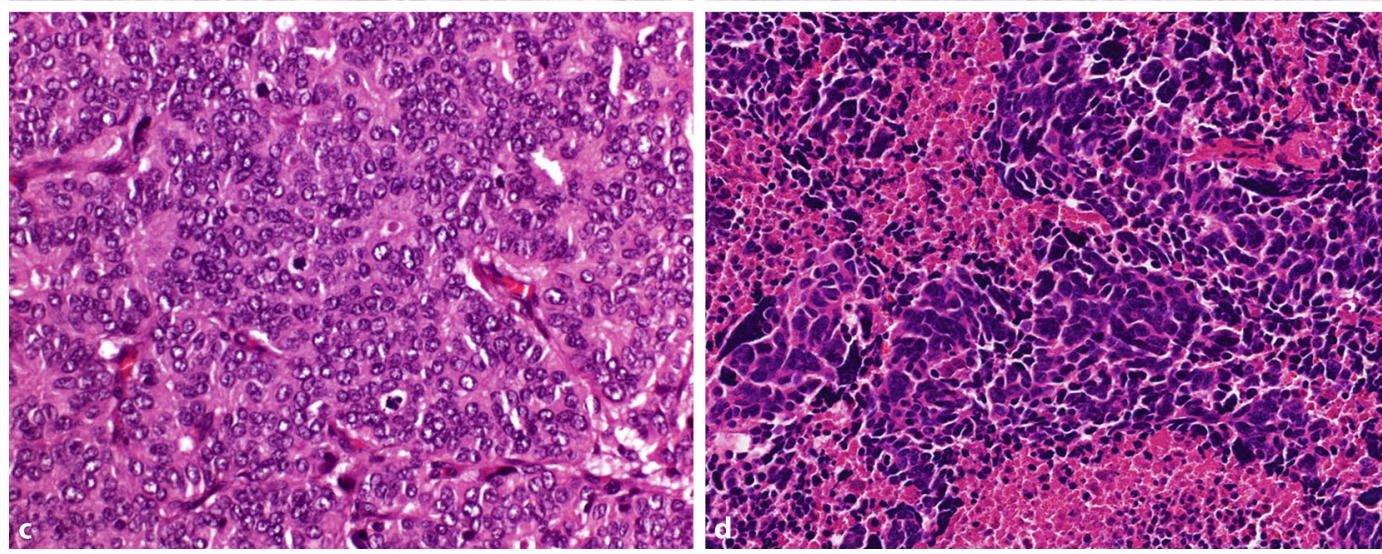
Wachstumsmuster und einigen Tumorzellen mit akzentuierten Atypien und Mitosen. Großzelliges neuroendokrines Karzinom (c) mit beinahe vollständig verlorenem organoidem Wachstum und gesteigerter Mitoserate. Kleinzelliges Karzinom (d) charakterisiert durch typisches zytologisches Erscheinungsbild der Tumorzellen, mit geringem Zytoplasma und ohne offensichtlichen Nukleoli sowie weitläufiger Nekrose

distinkt sind, allerdings in manchen Fällen immunhistochemischer Differenzierung bedürfen (Zytokeratin ist bei Paragangliomen negativ). Weiter müssen Typ-A-Thymome ausgeschlossen werden, die sich bei neuroendokrinen Markern negativ präsentieren. Typ-B3Thymome mit rosettenähnlichen Strukturen können ebenfalls eine schwierig zu differenzierende Diagnose darstellen. Muzinöse Varianten des typischen Karzinoids müssen hinsichtlich Adenokarzinomen des Gastrointestinaltrakts oder der Mamma ausgeschlossen werden, während amyloide Varianten (normalerweise Calcitonin-positiv) nicht von medullären Schilddrüsenkarzinomen unterschieden werden können. Selten präsentieren sich typische Karzinoide gemeinsam mit Thymomen und Thymuskarzinomen [18].

\section{Atypische Karzinoide}

Atypische Karzinoide sind definiert als neuroendokrine epitheliale Tumoren des Thymus, mit 2 bis 10 Mitosen $/ 2 \mathrm{~mm}^{2}$ (in $10 \mathrm{HPF}$ ) und/oder Nekrosen (• Abb. 2).
Sie treten häufiger als typische Karzinoide auf und machen etwa $50 \%$ aller NET des Thymus aus [26]. Wie typische Karzinoide treten auch sie häufiger bei Männern (bis zu $70 \%$ der Patienten) und im mittleren Alter auf (Durchschnittsalter 48 bis 55 Jahre, Altersspanne 18 bis 82 Jahre), wurden aber auch schon bei Kindern zwischen 8 und 16 Jahren beschrieben $[12,20]$. Atypische Karzinoide weisen in $50 \%$ der Fälle bereits zum Zeitpunkt der Diagnose Fernmetastasen auf [15, 24]. Meistens sind davon mediastinale, zervikale und supraklavikuläre Lymphknoten betroffen, wobei auch benachbarte Organe wie Pleura und Perikard infiltriert sein können. Fernmetastasen treten typischerweise in Lungen, Gehirn, Knochen, Leber und Nieren, aber auch in Nebennieren, Haut und Weichteilen auf [14, 24].

Histologisch und immunhistochemisch zeigen sich ähnliche Charakteristika wie für typische Karzinoide mit den Spezifikationen der höheren Mitoserate und/ oder Nekrosen, unabhängig von der Tumorgröße. Die Tumorzellen zeigen meist ein gröberes Chromatinmuster, Nukleo- len können mittelgroß sein, eine Polymorphie der Zellkerne ist nicht ungewöhnlich (• Abb. 1).

Die Differenzialdiagnosen sind identisch mit denen typischer Karzinoide (s. oben), wobei Paragangliome und Typ-A-Thymome die 2 wichtigsten zu differenzierenden Entitäten darstellen.

\section{Großzellige neuroendokrine Karzinome}

Großzellige neuroendokrine Tumoren des Thymus sind hochgradig maligne epitheliale Tumoren, die sich mit neuroendokriner Morphologie und Differenzierung präsentieren, welche über immunhistochemische oder elektronenmikroskopische Verfahren bestätigt werden können. Sie zeigen 11 oder mehr Mitosen $/ 2 \mathrm{~mm}^{2}$ (durchschnittlich $45 / 2 \mathrm{~mm}^{2}$ ) und regelmäßig extensive Koagulationsnekrosen. Insgesamt machen sie $14-26 \%$ der NET des Thymus aus (Inzidenz 1/20 Mio. [1, 25, 28]) und treten bei Männern doppelt so häufig auf wie bei Frauen. Die Altersspanne erstreckt sich von 16 bis 79 Jahren mit einem 


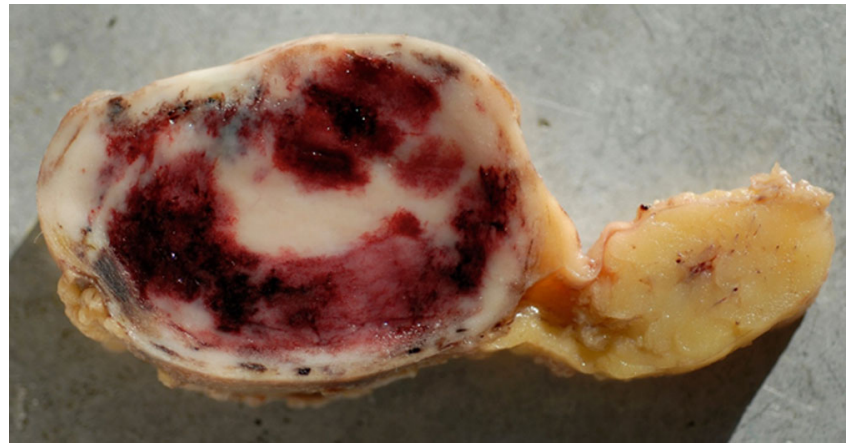

Abb. $2<$ Makroskopische Präsentation eines atypischen Karzinoids des Thymus. Offensichtliche Einblutungen und Nekrosezonen in makroskopisch weißer Tumormasse. Rechtsseitig mediastinales Fettgewebe

Median von 51 Jahren [5, 22, 25]. Bis zu $50 \%$ der Patienten präsentieren sich symptomlos, während die andere Hälfte ähnliche Symptomkonstellationen wie Patienten mit Karzinoiden aufweisen, namentlich Dyspnoe, Husten, Druckschmerz und Einflussstau. Das Auftreten eines Cushing-Syndroms ist selten und es existieren hierzu nur einzelne Fallberichte. Zum Zeitpunkt der Diagnose zeigen bereits $75 \%$ der Patienten Umgebungsinvasionen oder Fernmetastasen $[3,5,25]$.

\section{Histologie}

Histologisch können LCNEC atypischen Karzinoiden mit Ausnahme der obligat höheren Mitoserate sehr ähnlich sein. LCNEC zeigen allerdings häufig große Tumorzellen (teilweise sogar anaplastische Riesenzellen) und weniger offensichtlichen organoiden Strukturen, aber dafür mit landkartenartigen Nekrosen (- Abb. 1). Bei kleinen Biopsien kann die Differenzierung zwischen atypischem Karzinoid und großzellig neuroendokrinem Karzinom schwierig oder auch unmöglich sein: Die Mitoserate kann fokal sehr niedrig, die organoide Struktur gut ausgebildet sein. Erst im Operationspräparat lässt sich in manchen Fällen die ausreichend hohe Zahl von Mitosen nachweisen, die für die Diagnose eines LCNEC erforderlich ist.

\section{Immunhistochemie}

Immunhistochemisch sind LCNEC Karzinoiden sehr ähnlich bzgl. Expression der Zytokeratine AE1/AE3 sowie CAM 5.2 und neuroendokrinen Marker (CD56, Synaptophysin, Chromogranin, NSE). Definitionsgemäß wird mindestens einer der neuroendokrinen Marker in mehr als $50 \%$ der Tumorzellen ex- primiert. Teilweise kann CD117 positiv sein, wobei CD5 immer negativ ist [5, 8].

\section{Differenzialdiagnostik}

Bei in seltenen Fällen vorhandener TTF1Positivität muss ein primär hochgradig maligner NET der Lunge durch eine detaillierte klinische und radiologische Analyse ausgeschlossen werden. Thymuskarzinome können ebenso neuroendokrine Marker exprimieren [17, 19], wobei diese Reaktionen nur fokal und schwach auftreten, was die Differenzierung $\mathrm{zu}$ großzelligen neuroendokrinen Karzinomen ermöglicht, bei denen neuroendokrine Marker stark positiv sind und mehr als $50 \%$ der Tumorzellen positiv reagieren.

\section{Kleinzellige Karzinome}

Kleinzellige Karzinome (SCC) des Thymus stellen hochgradige epitheliale Tumoren des Thymus dar, die durch solide Nester und diffuses Wachstum ovaler, runder und Spindelzellen, mit schmalem Zytoplasma, fein granulierten Nuklei und nicht vorhandenen oder unauffälligen Nukleoli charakterisiert sind. Weitläufige Nekrosen, eine hohe Mitoserate und Kernmorphologie sind typisch. Nur $10 \%$ aller NET des Thymus sind kleinzellige Karzinome (Inzidenz 1/50 Mio. $[11,25,28])$. Das Mediastinum mit seinen Lymphknoten ist häufig Sitz von Metastasen eines primären Lungenkarzinoms, und die Unterscheidung zwischen primärem mediastinalem kleinzelligem Karzinom und der Metastase eines pulmonalen Typs kann schwierig sein. Interessanterweise gibt es keinen Zusammenhang zwischen Rauchen und diesen Karzinomen. Kleinzellige Karzino- me sind aggressive Tumoren, die meisten Patienten präsentieren sich sowohl mit lokalen Symptomen als auch Infiltration benachbarter Organe (Lungen, Perikard und Pulmonalarterien) sowie Fernmetastasen (Lungen, Knochen, Gehirn, Leber, abdominelle Lymphknoten [25, 28, 31]).

\section{Histologie}

Histologisch präsentieren SCC sich wie kleinzellige Karzinome in anderen Lokalisationen, mit einer Tumorzellgröße entsprechend 2 bis 3 ruhenden Lymphozyten, schmalem Zytoplasma, unscheinbaren Nukleoli sowie hohen Mitoseraten (durchschnittlich 110/2 $\mathrm{mm}^{2}$; [- Abb. 1]). Die meisten thymischen kleinzelligen Karzinome sind für die vorhin beschriebenen neuroendokrinen Marker positiv, aber in seltenen Fällen kann eine neuroendokrine Differenzierung gänzlich fehlen. Zytokeratine färben die meisten, aber nicht alle Tumoren. TTF1-Reaktionen sind bisher noch nicht systematisch analysiert worden.

\section{Differenzialdiagnostik}

Die Differenzialdiagnostik richtet sich primär an kleinzellige Lungen-Primärherde, nachdem primär mediastinale kleinzellige Karzinome sehr selten sind. Eine detaillierte radiologische und chirurgische Analyse ist für die Unterscheidung notwendig. In zytokeratinnegativen Fällen müssen immer erst nichtepitheliale Malignome, wie T-lymphoblastische Lymphome und primitive neuroektodermale Tumore mittels zusätzlichen immunhistochemischen Färbungen wie CD45, CD3, CD99 und/oder durch andere Methoden wie FISH (für EwingTumoren) ausgeschlossen werden.

\section{Genetische Unterschiede}

Interessanterweise zeigen bis zu $40 \%$ der typischen Karzinoide keine Alteration in der komparativen genomischen Hybridisierung (CGH). In den übrigen Fällen finden sich Veränderungen wie beispielsweise Zugewinne an den Chromosomen 1, 2q24, 7, 8p, 8q, 9q13, 11q23qter und 22 sowie Verluste an 1p, 3p11, 6q, $10 q$ und 13q. Atypische Karzinoide zeigen Gewinne an 1q, 5p, 5q, 7p, 7q, 8q, 
$12 q 24,17 q$ und $20 q$ sowie Verluste an $3 p$, $3 \mathrm{q}, 4 \mathrm{q}, 5 \mathrm{q}, 6 \mathrm{q}, 10 \mathrm{q}, 11 \mathrm{q} \mathrm{q}$ und $13 \mathrm{q}$. Obwohl bei $8 \%$ der Patienten mit MEN1Syndrom Karzinoide des Thymus auftreten, ist das MEN1-Gen auf Chromosom 11q13 in diesen Tumoren nicht verändert. LCNEC zeigen teilweise dieselben genetischen Alterationen wie Karzinoide (Zugewinne an 1q, 6p,7, 8q, 12q und 14 sowie Verluste an 3, 4q und 13q), aber in einer höheren Frequenz. Molekulare Veränderungen, die nur in großzelligen neuroendokrinen Karzinomen gefunden werden können, umfassen Zugewinne auf $2 p, 9 p$ und $17 q$ sowie Verluste an $4 p, 8 p$, $9 p$ und 18p. Bezüglich kleinzelliger Karzinome liegen derzeit keine suffizienten Daten vor, allerdings finden sich chromosomale Überlappungen mit Karzinoiden und LCNEC. Die genetischen Profile typischer und atypischer Karzinoide des Thymus unterscheiden sich von denen der pulmonalen Karzinoide, während großzellige neuroendokrine Karzinome und kleinzellige Karzinome dieselben Veränderungen wie bei Auftreten in der Lunge aufweisen [25].

\section{Therapie}

Relevante Publikationen zu den Themen Therapie und Prognose sind spärlich. Beide sind abhängig vom TNM- bzw. Masaoka-Koga-Stadium (wobei beide Klassifikationen weiterhin inoffiziell und zum Staging der mediastinalen NET nicht universell akzeptiert sind). Die Therapie der Wahl stellt die chirurgische Exzision inklusive regionaler Lymphknotenentfernung dar (wobei letztere ebenso noch nicht standardisiert praktiziert wird). Bezüglich Chemo- und/oder Radiotherapie liegen derzeit keine adäquaten $\mathrm{Da}$ ten vor, wobei adjuvante Therapien in inoperablen Fällen jedenfalls zu erwägen sind. Manche Autoren sprechen sich für eine adjuvante Chemo-, Radio- oder Radiochemotherapie für Patienten mit hochgradigen NET des Thymus in jedem Stadium aus. Aufgrund der Expression von Somatostatinrezeptoren wurden auch Somatostatinanaloga (Octreotid, Lanretid) alleine oder in Kombination mit $\alpha$-Interferon oder Tyrosinkinaserezeptorinhibitoren eingesetzt $[7,9,10$,
26, 27], jedoch ohne signifikante Ergebnisse zu erzielen.

\section{Prognose}

Die Prognose hängt außer vom Tumorstadium auch vom -grading ab. Das 5-Jahres-Überleben liegt bei typischen Karzinoiden zwischen 50-70\% [24, 25] und somit geringfügig höher als für atypische Karzinoide mit einer Rate von 20-80\% [6, 15, 24, 25], wobei letztere auch zu Rezidivierung neigen. Patienten, die ein Cushing-Syndrom entwickeln, haben eine besonders schlechte Prognose. Außerdem weisen NET des Thymus, verglichen mit NET anderer Organe, generell eine schlechtere Prognose auf [32]. Die publizierten 5-Jahres-Überlebensraten für LCNEC belaufen sich auf 30-66 \% $[3,21,25,28]$, womit sie praktisch identisch sind mit denen der atypischen Karzinoide, während kleinzellige Karzinome mit einem durchschnittlichen Überleben von 13 bis 26 Monaten die schlechteste Prognose aufweisen [25, 28].

\section{Fazit für die Praxis}

- NET des Mediastinums sind selten und machen etwa $5 \%$ aller mediastinalen Tumoren aus. Unter ihnen sind atypische Karzinoide am häufigsten.

- NET des Thymus können sich einerseits als kleine und praktisch immer funktionelle Tumoren präsentieren, aber auch bis zu $20 \mathrm{~cm}$ groß werden. Etwa zwei Drittel sind durch lokales Tumorwachstum, Fernmetastasen und/oder endokrine Symptomatik symptomatisch.

- Die Klassifizierung basiert auf der Mitoserate und dem Vorliegen von Nekrosen, wodurch sich eine Unterscheidung in niedrig-, mäßig- und hochgradig maligne NET ergibt. Zur Differenzialdiagnostik sind neuroendokrine Marker oft unumgänglich. Ausgenommen davon sind kleinzellige Karzinome, bei denen diese Marker nicht ausschlaggebend für die Diagnose sind.

- Staging und Therapie sind noch nicht standardisiert, wobei letztere durchgängig durch Tumorexzision erfolgt. Die Prognose ist abhängig vom Malignitätsgrad und somit für kleinzellige Karzinome am schlechtesten.

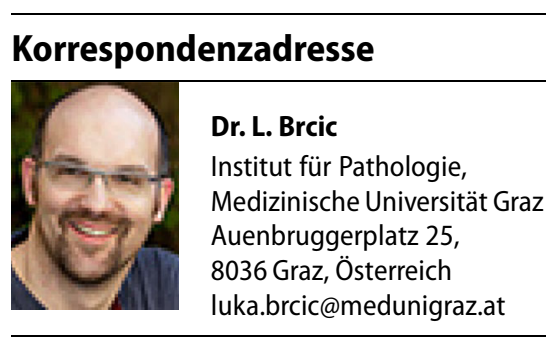

Open access funding provided by Medical University of Graz.

\section{Einhaltung ethischer Richtlinien}

Interessenkonflikt. L. Brcic, M. Heidinger und H. Popper geben an, dass kein Interessenkonflikt besteht.

Dieser Beitrag beinhaltet keine von den Autoren durchgeführten Studien an Menschen oder Tieren.

Open Access Dieser Artikel wird unter der Creative Commons Namensnennung 4.0 International Lizenz (http://creativecommons.org/licenses/by/4.0/deed. de) veröffentlicht, welche die Nutzung, Vervielfältigung, Bearbeitung, Verbreitung und Wiedergabe in jeglichem Medium und Format erlaubt, sofern Sie den/die ursprünglichen Autor(en) und die Quelle ordnungsgemäßnennen, einen Linkzur Creative Commons Lizenz beifügen und angeben, ob Änderungen vorgenommen wurden.

\section{Literatur}

1. Ahn S, Lee JJ, Ha SY et al (2012) Clinicopathological analysis of 21 thymic neuroendocrine tumors. J Pathol Transl Med 46(3):221-225. doi:10.4132/ KoreanJPathol.2012.46.3.221

2. Axelson J, Kobari M, Furukawa T, Matsuno $S$ (1999) Thymic carcinoid in the pancreas: metastatic disease or new primary tumours. Eur J Surg 165(3):270-273. doi:10.1080/ 110241599750007171

3. Cardillo G, Treggiari S, Paul MA et al (2010) Primary neuroendocrine tumours of the thymus: a clinicopathologic and prognostic study in 19 patients. Eur J Cardiothorac Surg 37(4):814-818. doi:10.1016/j.ejcts.2009.10.026

4. Chaer R, Massad MG, Evans A et al (2002) Primary neuroendocrine tumors of the thymus. Ann Thorac Surg 74(5):1733-1740. doi:10.1016/s00034975(02)03547-6

5. Chetty R, Batitang S, Govender D (1997) Large cell neuroendocrine carcinoma of the thymus. Histopathology 31(3):274-276. doi:10.1046/j. 1365-2559.1997.2380849.x

6. Crona J, Bjorklund P, Welin S et al (2013) Treatment, prognostic markers and survival in thymic neuroendocrine tumours. a study from a single tertiary referral centre. Lung Cancer 79(3):289-293. doi:10.1016/j.lungcan.2012.12. 001 
7. Dham A, Truskinovsky AM, Dudek AZ (2008) Thymic carcinoid responds to neoadjuvant therapy with sunitinib and octreotide: a case report. J Thorac Oncol 3(1):94-97. doi:10.1097/ JT0.0b013e31815eb7a2

8. Dutta R, Kumar A, Julka PK et al (2010) Thymic neuroendocrine tumour (carcinoid): clinicopathological features of four patients with different presentation. Interact Cardiovasc Thorac Surg 11(6):732-736. doi:10.1510/icvts.2010.237651

9. Ferolla P, Falchetti A, Filosso P et al (2005) Thymic neuroendocrine carcinoma (carcinoid) in multiple endocrine neoplasia type 1 syndrome: the Italian series. J Clin Endocrinol Metab 90(5):2603-2609. doi:10.1210/jc.2004-1155

10. Filosso PL, Actis Dato GM, Ruffini E et al (2004) Multidisciplinary treatment of advanced thymic neuroendocrine carcinoma (carcinoid): report of a successfulcase and review of the literature.JThorac Cardiovasc Surg 127(4):1215-1219. doi:10.1016/j. jtcvs.2003.09.058

11. Gal AA, Kornstein MJ, Cohen C et al (2001) Neuroendocrine tumors of the thymus: a clinicopathological and prognostic study. Ann Thorac Surg 72(4):1179-1182. doi:10.1016/s0003 4975(01)03032-6

12. Gartner LA, Voorhess ML (1993) Adrenocorticotropic hormone - producing thymic carcinoid in a teenager. Cancer 71(1):106-111. doi:10.1002/ 1097-0142(19930101)71:13.0.co;2-u

13. Gaur P, Leary C, Yao JC (2010) Thymic neuroendocrine tumors: a SEER database analysis of 160 patients. Ann Surg 251(6):1117-1121. doi:10. 1097/SLA.0b013e3181dd4ec4

14. Gibril F, Chen Y-J, Schrump DS et al (2003) Prospective study of thymic carcinoids in patients with multiple endocrine neoplasia type 1. J Clin Endocrinol Metab 88(3):1066-1081. doi:10.1210/ jc.2002-021314

15. Goto K, Kodama T, Matsuno Yetal (2001) Clinicopathologic and DNA cytometric analysis of carcinoid tumors of the thymus. ModPathol 14(10):985-994. doi:10.1038/modpathol.3880423

16. Goudet P, Murat A, Cardot-Bauters C et al (2009) Thymic neuroendocrine tumors in multiple endocrine neoplasia type 1: a comparative study on 21 cases among a series of 761 MEN1 from the GTE (Groupe des Tumeurs Endocrines). World J Surg 33(6):1197-1207. doi:10.1007/s00268-0099980-y

17. Hishima T, Fukayama M, Hayashi Y et al (1998) Neuroendocrine differentiation in thymic epithelial tumors with special reference to thymic carcinoma and atypical thymoma. Hum Pathol 29(4):330-338. doi:10.1016/s0046-8177(98)90112-5

18. Kuo TT, Chang JP, Lin FJ et al (1990) Thymic carcinomas: histopathological varieties and immunohistochemical study. Am J Surg Pathol 14(1):24-34. doi:10.1097/00000478-19900100000003

19. Lauriola L, ErlandsonRA, Rosai J(1998) Neuroendocrine differentiation is a common feature of thymic carcinoma. Am J Surg Pathol 22(9):1059-1066. doi:10.1097/00000478-199809000-00003

20. Lin KL, Chen CY, Hsu HH et al (1999) Ectopic ACTH syndrome due to thymic carcinoid tumor in a girl. J Pediatr Endocrinol Metab 12(4):573-578. doi:10. 1515/jpem.1999.12.4.573

21. Mega S, Oguri M, Kawasaki R et al (2008) Largecell neuroendocrine carcinoma in the thymus. Gen Thorac Cardiovasc Surg 56(11):566-569. doi:10. 1007/s11748-008-0299-7

22. Ogawa F, lyoda A, Amano H et al (2010) Thymic large cell neuroendocrine carcinoma: report of a resected case - a case report. J Cardiothorac Surg 5(1):115. doi:10.1186/1749-8090-5-115

23. Oliveira AM, Tazelaar HD, Myers JL et al (2001) Thyroid transcription factor-1 distinguishes metastatic pulmonary from well-differentiated neuroendocrine tumors of other sites. Am J Surg Pathol 25(6):815-819. doi:10.1097/00000478200106000-00015

24. Soga J, Yakuwa Y, Osaka M (1999) Evaluation of 342 cases of mediastinal/thymic carcinoids collected from literature: a comparative study between typical carcinoids and atypical varieties. Ann Thorac Cardiovasc Surg 5(5):285-292

25. Strobel P, Zettl A, Shilo K et al (2014) Tumor genetics and survival of thymic neuroendocrine neoplasms: a multi-institutional clinicopathologic study. Genes Chromosomes Cancer 53(9):738-749. doi:10.1002/gcc.22183

26. Teh BT (1998) Thymic carcinoids in multiple endocrine neoplasia type 1. J Intern Med 243(6):501-504. doi:10.1046/j.1365-2796.1998. 00329.x

27. Teh BT, McArdle J, Chan SP et al (1997) Clinicopathologic studies of thymic carcinoids in multiple endocrine neoplasia type 1. Medicine (Baltimore) 76(1):21-29. doi:10.1097/00005792-19970100000002

28. Tiffet O, Nicholson AG, Ladas G et al (2003) A clinicopathologic study of 12 neuroendocrine tumors arising in the thymus. Chest 124(1):141-146. doi:10.1378/chest.124.1.141

29. Travis WD, Brambilla E, Burke AP, Marx A, Nicholson AG (2015) WHO classification of tumours of lung, pleura, thymus and heart, 4. Aufl. IARC, Lyon

30. Travis WD, BrambillaE, Muller-HermelinkHK, Harris CC (2004) World Health Organization classification of tumours. Pathology and genetics of tumours of the lung, pleura, thymus and heart. IARC, Lyon

31. Truong LD, Mody DR, Cagle PT et al (1990) Thymic carcinoma. A clinicopathologic study of 13 cases. Am J Surg Pathol 14(2):151-166. doi:10.1097/ 00000478-199002000-00007

32. Wick MR, Carney JA, Bernatz PE, Brown LR (1982) Primary mediastinal carcinoid tumors. Am J Surg Pathol 6(3):195-205. doi:10.1097/00000478198204000-00001

33. Yoshikawa T, Noguchi Y, Matsukawa H et al (1994) Thymus carcinoid producing parathyroid hormone (PTH)-related protein: report of a case. Surg Today 24(6):544-547.doi:10.1007/bf01884576

\section{Protokoll zur Metaboliten- analyse in fixiertem Gewebe}

Wissenschaftler am Helmholtz Zentrum München haben eine neue Methode für die bildgebende Massenspektrometrie entwickelt, die es erstmals ermögliche, in fixierten Gewebeproben hunderte von Metaboliten gleichzeitig zu analysieren. Dazu reichen relativ kleine Mengen an Material, sodass dies auch die Analyse von kleinsten Biopsien und sogar Gewebe-Mikroarrays erlaube. Um auszuschließen, dass die gemessenen Daten nicht durch den Fixationsprozess verfälscht werden, verglichen die Autoren diese mit Messwerten der gleichen Proben, die aber nicht fixiert, sondern schockgefroren waren. Dabei habe sich ein Großteil der gemessenen Metabolite in beiden Analysen wiedergefunden. Dies zeige, dass die Methode verlässlich funktioniert und dabei die aufwändige Logistik und Lagerung von schockgefrorenen Proben umgeht, erklären die Forscher. Neben der einfachen Handhabung und der hohen Reproduzierbarkeit sei auch die Möglichkeit, mit hohem Probendurchsatz zu arbeiten, ein wichtiger Vorteil der neuen Methode. Vor allem aber könne man nun die räumliche Verteilung von Molekülen im Gewebe bildhaft und mit großer Präzision studieren. Ziel sei es nun, mit dem neuen Analyseverfahren zukünftig neue prädiktive, diagnostische und prognostische Marker in Geweben zu identifizieren, sowie Krankheitsprozesse besser zu verstehen.

\section{Quelle: Helmholtz Zentrum München www.helmholtz-muenchen.de}

basierend auf: Nature Protocols (2016)

DOI: nprot.2016.081 